\title{
GAMMA-RAY IMAGING OF THE GALACTIC CENTER REGION
}

\author{
W. R. COOK, D. M. PALMER, T. A. PRINCE, S. M. SCHINDLER, \\ C. H. STARR, AND E. C. STONE \\ California Institute of Technology \\ Pasadena, CA 91125
}

\begin{abstract}
The Caltech imaging $\gamma$-ray telescope was launched by balloon from Alice Springs, NT, Australia and performed observations of the galactic center during the period 12.62 to 13.00 April 1988 UT. The first coded-aperture images of the galactic center region at energies above 30 $\mathrm{keV}$ show a single strong $\gamma$-ray source which is located $0.7 \pm 0.1^{\circ}$ from the galactic nucleus and is tentatively identified as 1E1740.7-2942. If the source is at the distance of the galactic center, it is one of the most luminous objects in the galaxy at energies from 35 to $200 \mathrm{keV}$.
\end{abstract}

\section{Introduction}

Hard X-ray and $\gamma$-ray observations of the galactic center region have been performed by balloon-borne and spacecraft instruments since the early 1970 s. $^{1,2,3,4}$ However, only recently has imaging been performed in the hard X-ray energy range - the Spacelab 2 observations of Skinner et al. ${ }^{5}$ from 3 to $30 \mathrm{keV}$. These results, together with the observations performed with a slit scanning experiment on Spartan 1 from 1 to 15 $\mathrm{keV}^{6}$ and the Einstein observatory from 0.9 to $4 \mathrm{keV},{ }^{7}$ have revealed a number of point sources within approximately one degree of the galactic nucleus, including a point source at the nucleus itself surrounded by a region of diffuse emission.

Non-imaging observations at higher energies have revealed $0.511 \mathrm{MeV}$ positron annihilation line radiation and hard continuum emission extending to above $1 \mathrm{MeV}$, with both line and continuum components varying in intensity on time scales as short as six months. $8,9,10$ The compact source size required by the time variability, and the unusual $\gamma$-ray spectrum, have stimulated speculation that the emission may be due to a massive black hole at the galactic nucleus. Although the location of the $0.511 \mathrm{MeV}$ source is consistent with this speculation, the observational uncertainty of $\pm 4^{\circ}$ prevents a definitive identification.

We report here the identification of a strong source of $\gamma$-rays based on the first coded-aperture imaging observation of the galactic center region at energies above 30 $\mathrm{keV}$. However, the source is not at the galactic nucleus, and $0.511 \mathrm{MeV}$ line and hard $\mathrm{MeV}$ continuum emissions were not observed.

\section{Observations}

The observations were performed with the Caltech Gamma-Ray Imaging Payload (GRIP), a balloon-borne coded-aperture telescope sensitive to radiation in the energy range from $30 \mathrm{keV}$ to $10 \mathrm{MeV}$. ${ }^{1 \mathrm{~T}}$ The instrument employs a rotating hexagonal-celled uniformly redundant array (URA) and a $5 \mathrm{~cm}$ thick by $41 \mathrm{~cm}$ diameter position sensitive $\mathrm{NaI}$ scintillation detector to image a $14^{\circ}$ diameter field of view with $1.1^{\circ}$ angular resolution. 
The $\gamma$-ray telescope is mounted in an elevation-azimuth pointing gondola. Telescope elevation is controlled by a stepping motor driving a ball-screw mechanism, while azimuth is controlled by a torque motor employing magnetometer feedback. Redundant magnetometer systems provide aspect information used in post-flight analysis to correct for azimuthal tracking errors. In addition, during the flight reported here, improved aspect information was obtained from: (1) a CCD camera which could track the sun, planets or bright stars using a mirror mounted on a precision rotation stage, and (2) an image-intensified CCD star camera sensitive down to 7 th magnitude.

In April 1988, a 30 hour flight of the instrument was achieved, and the galactic center region was observed for two 4-hour periods during the interval 12.62 to 13.00 April 1988 UT. Data from the star camera has been used in a preliminary analysis of one hour of the galactic center observation extending from 12.751 to 12.792 April 1988 UT. The resulting image, shown in Figure $1 a$ for the 35 to $200 \mathrm{keV}$ energy interval, contains a single significant image peak. Known hard X-ray sources are indicated by dots. ${ }^{5,12}$ An expanded view of the image peak of Figure $1 a$ is shown in Figure $1 b$. The location of the peak, indicated by a cross, is within $0.1^{\circ}$ of source number $5,1 \mathrm{E} 1740.7$ 2942. Our estimated $90 \%$ confidence error box for the peak location excludes all other hard X-ray sources shown, although source number 8, A1742-294, is near one corner of the box. The galactic nucleus source Sgr A* (source number 7 ) is $0.7 \pm 0.1^{\circ}$ away from the image peak, and well outside the error box.

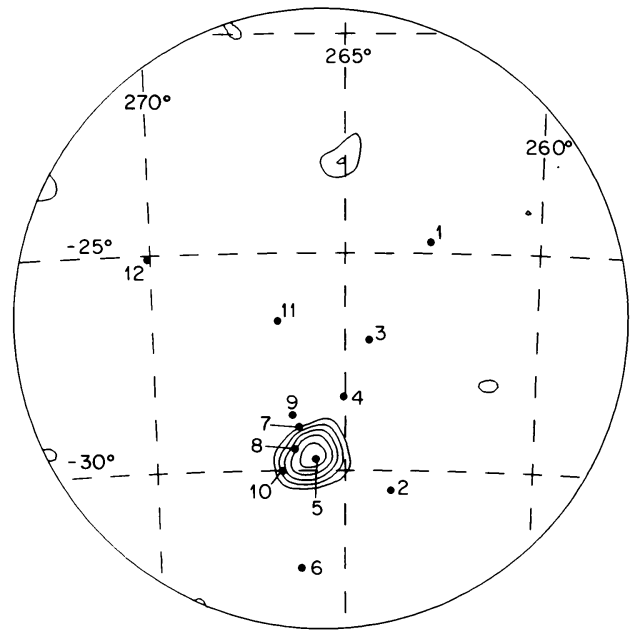

(a)

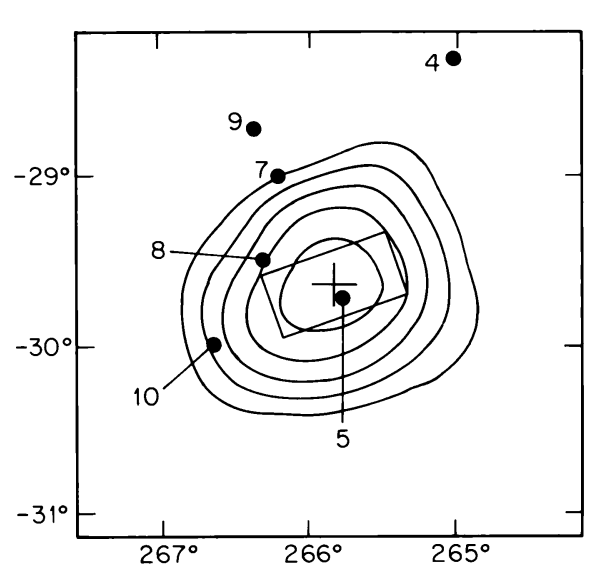

(b)

Fig. 1.-(a) Image of the galactic center region from 35 to $200 \mathrm{keV}$ covering a $14^{\circ}$ field of view. Right ascension (vertical lines) and declination (horizontal lines) are indicated for epoch 1988.3. The contours indicate the number of excess counts in a given direction, calibrated in units of the statistical significance of the excess, with contours beginning at the $2 \sigma$ level and spaced by $1 \sigma$. Known hard X-ray sources, ${ }^{5}$ are: (1) GX1+4, (2) SLX1732-304, (3) SLX1735-269, (4) SLX1737282 , (5) 1E1740.7-2942, (6) A1743-322, (7) Sgr A*, (8) A1742-294, (9) 1E1743.1-2843, (10) SLX1744-299, (11) GX3+1, and (12) GX5-1. (b) Expanded view of the image peak. The peak location and its $90 \%$ confidence error box are indicated. 
The estimated error box dimensions include both statistical and possible systematic uncertainties. The error box is narrower along one axis due to use of the star camera aspect data. The camera provides accurate aspect information only along the arc connecting the axis of the $\gamma$-ray telescope with that of the star camera, which is offset by $50^{\circ}$. The longer dimension of the error box aligns with the direction of telescope elevation motion. The size of the error box in this dimension was estimated based on elevation pointing errors measured in seven pointings at the Crab nebula and pulsar and a single pointing at $\mathrm{Cyg} \mathrm{X}-1$.

To obtain a preliminary energy spectrum for the source seen in Figure 1, the remainder of the galactic center observation was processed in one hour segments without the use of star camera data. A single image peak similar to that of Figure 1 was seen in the 35 to $200 \mathrm{keV}$ image for each of the additional time periods. Pointing offsets were chosen to align each image peak with that of Figure 1. A composite 35 to $200 \mathrm{keV}$ image for all the time periods was found to again have only a single significant $(16 \sigma)$ image peak, of shape consistent with the point source response measured for the Crab and Cyg X1. Thus we have assumed a single point source and derived its photon energy spectrum by analysis of images for a series of energy bands from $35 \mathrm{keV}$ to above $6 \mathrm{MeV}$. The resulting source spectrum is shown in Figure 2. A minimum chisquared fit to a power law function,

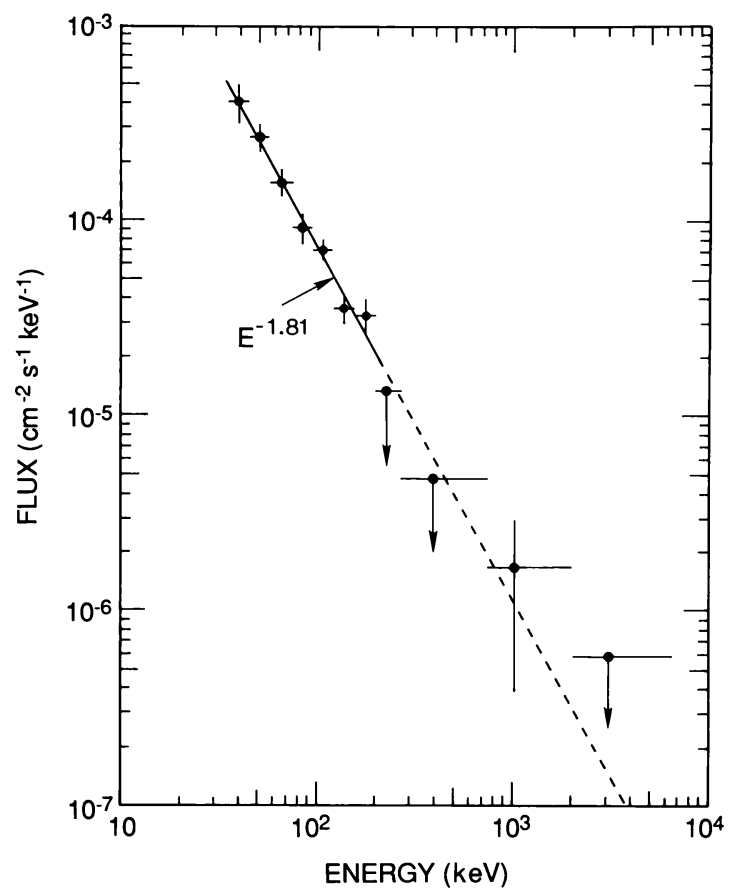

Fig. 2.-Gamma-ray differential energy spectrum for the source shown in Fig. 1. Upper limits are $2 \sigma$. $d J / d E=K(E / 100 \mathrm{keV})^{\alpha}$, was performed for the energy range from 35 to $200 \mathrm{keV}$. The best fit power law spectrum, shown in Figure 2, has a spectral index $\alpha=-1.81 \pm 0.12$ and a flux normalization $K=7.4 \pm 0.5 \times 10^{-5} \mathrm{~cm}^{-2} \mathrm{~s}^{-1} \mathrm{keV}^{-1}$. A similar analysis for the Crab nebula and pulsar from 35 to $173 \mathrm{keV}$ gave $\alpha=-1.80 \pm 0.06$ and $K=4.7 \pm 0.1 \times 10^{-4}$ $\mathrm{cm}^{-2} \mathrm{~s}^{-1} \mathrm{keV}^{-1}$.

An image has also been generated for a $55 \mathrm{keV}$ wide energy band centered on 0.511 $\mathrm{MeV}$, and chosen to include $89 \%$ of the flux from a narrow line. No significant image peak was found. The preliminary $99 \%$ confidence upper limit for $0.511 \mathrm{MeV}$ line flux from a point source at the position of the hard X-ray image peak is $8 \times 10^{-4} \mathrm{~cm}^{-2} \mathrm{~s}^{-1}$.

The results reported here are preliminary. We note that the Crab spectrum given above is $\sim 5-35 \%$ below other measurements at $100 \mathrm{keV},{ }^{13}$ and may indicate an overall systematic underestimation of the flux. However, the earlier measurements were made with non-imaging instruments which may overestimate the flux, and one cannot exclude the possibility of time variability. 


\section{Discussion}

The first coded-aperture imaging observation of the galactic center region at energies above $30 \mathrm{keV}$ has revealed a single (at $1.1^{\circ}$ resolution) strong source of hard X-rays and $\gamma$-rays. If the source is at a distance of $8.5 \mathrm{kpc}$, its 35 to $200 \mathrm{keV}$ luminosity of $1.7 \times 10^{37} \mathrm{ergs} / \mathrm{s}$ would be one of the largest in the galaxy, comparable to the 50 to 400 $\mathrm{keV}$ luminosity of $2.3 \times 10^{37} \mathrm{ergs} / \mathrm{s}$ measured for the black hole candidate Cyg X-1 in its $\gamma_{3}$ state. $^{14}$

Our observation of a single hard X-ray image peak at energies above $35 \mathrm{keV}$ is in good accord with the earlier imaging results from Spacelab 2 at energies below $30 \mathrm{keV}$. The Spacelab 2 results show that only one source, 1E1740.7-2942 (number 5), dominates in the 19 to $30 \mathrm{keV}$ interval. A preliminary Spacelab 2 spectrum ${ }^{15}$ obtained for 1E1740.7-2942 during August 1985 connects smoothly with the spectrum shown in Figure 2, matching in both intensity $\left(\sim 7 \times 10^{-4} \mathrm{~cm}^{-2} \mathrm{~s}^{-1} \mathrm{keV}^{-1}\right.$ at $\left.30 \mathrm{keV}\right)$ and slope. Thus, both positional and spectral agreement suggest that the hard X-ray source seen in Figure 1 is $1 E 1740.7-2942$. The spectral agreement also suggests that the source spectrum may be relatively stable with time.

Our results should also be compared with previous observations of the galactic center region obtained with non-imaging and scanning instruments. Since such comparisons are complicated by differences in angular resolution and the known time variability of many of the hard X-ray sources in the region, we restrict ourselves to the following comments:

(1) The spectrum of Figure 2 falls below or near the lower envelope of previous results obtained at energies from about $50 \mathrm{keV}$ to $1 \mathrm{MeV}$ with wide aperture $\left(>10^{\circ} \mathrm{FWHM}\right)$ instruments. ${ }^{4}$ The higher results obtained with wide aperture instruments are, at energies below $100 \mathrm{keV}$, probably due to the inclusion of several (typically time-variable) hard X-ray sources located within $10^{\circ}$ of the galactic center.

(2) Several instruments ${ }^{4,12,16,17}$ with narrower apertures $\left(1.5^{\circ}\right.$ to $\left.5^{\circ} \mathrm{FWHM}\right)$ have observed a source near the galactic center with flux at $100 \mathrm{keV}$ ranging from $\sim 0.5$ to $2.0 \times 10^{-4} \mathrm{~cm}^{-2} \mathrm{~s}^{-1} \mathrm{keV}^{-1}$, comparable to that shown in Figure 2. Our observations support the suggestion by Skinner et al. ${ }^{5}$, that $1 \mathrm{E} 1740.7-2942$ is the most probable source for the high energy flux seen in these earlier observations.

(3) We have found no evidence for the reappearance of the compact source of hard $\mathrm{MeV}$ continuum radiation, which was seen to turn off or decrease between the HEAO 3 observations of Fall 1979 and Spring $1980 .^{10}$ The flux decrease was most dramatic at energies near $1 \mathrm{MeV}$, where the continuum level dropped by a factor of 20 or more. The spectrum of Figure 2 is a factor of two or more below the HEAO 3 Fall 1979 spectrum near $1 \mathrm{MeV}$. Further, our $0.511 \mathrm{MeV}$ upper limit of $8 \times 10^{-4} \mathrm{~cm}^{-2} \mathrm{~s}^{-1}$ is significantly below the value of $1.85 \pm 0.21 \times 10^{-3} \mathrm{~cm}^{-2} \mathrm{~s}^{-1}$ reported for Fall 1979. ${ }^{9}$ Our $0.511 \mathrm{MeV}$ upper limit is also lower than the reported decrease of $1.20 \pm 0.35 \times 10^{-3} \mathrm{~cm}^{-2} \mathrm{~s}^{-1}$ between Fall 1979 and Spring 1980, attributed to a compact source. However, the spectrum of Figure 2 and the $0.511 \mathrm{MeV}$ upper limit were derived for a single point in our field of view. Upper limits on the $0.511 \mathrm{MeV}$ line and $\mathrm{MeV}$ continuum from a possible point source at an arbitrary location in our field of view, and from diffuse emission, must await further analysis. 


\section{Acknowledgements}

We acknowledge the important contributions to the development of the GRIP telescope made by W. Althouse, D. Burke, A. Cummings, M. Finger, J. Weger and the personnel of the Central Engineering Services at Caltech. We thank the personnel of the National Scientific Balloon Facility and the NASA Wallops Flight Facility for their excellent balloon launch support. This work was supported by NASA grant NGR 05002-160.

\section{References}

(1) Leventhal, M. 1987, in 13th Texas Symposium on Relativistic Astrophysics, edited by M. Ulmer, 382.

(2) Lingenfelter, R. E., and Ramaty, R. 1988, review in these proceedings.

(3) Skinner, G. K., review in these proceedings.

(4) Matteson, J. L. 1982, in The Galactic Center, edited by G. R. Riegler and R. D. Blandford, AIP Conf. Proc. No. 83, 109.

(5) Skinner, G. K. et al. 1987, Nature, 330, 544.

(6) Kawai, N. et al. 1988, Ap. J., 330, 130.

(7) Watson, M. G., Willingale, R., Grindlay, J. E., and Hertz, P. 1981, Ap. J., 250, 142.

(8) Leventhal, M., MacCallum, C. J., Huters, A. F., and Stang, P. D. 1982, Ap. J., 260, L1.

(9) Riegler, G. R. et al. 1981, Ap. J., 248, L13.

(10) Riegler, G. R. et al. 1985, Ap. J., 294, L13.

(11) Althouse, W. E. et al. 1985, Proc. 19th Int. Cosmic Ray Conf. (La Jolla), 3, 299.

(12) Levine, A. M. et al. 1984, Ap. J. Suppl., 54, 581.

(13) Jung, G. V. 1986, Ph.D. thesis, University of California at San Diego.

(14) Ling, J. C., Mahoney, W. A., Wheaton, Wm. A., and Jacobson, A. S. 1987, Ap. J., 321, L117.

(15) Skinner, G. K., poster paper this conference.

(16) Knight, K. F., Johnson III, W. N., Kurfess, J. D., and Strickman, M. S. 1985, Ap. J., 290, 557.

(17) Dennis, B. R. et al. 1980, Ap. J., 236, L49. 